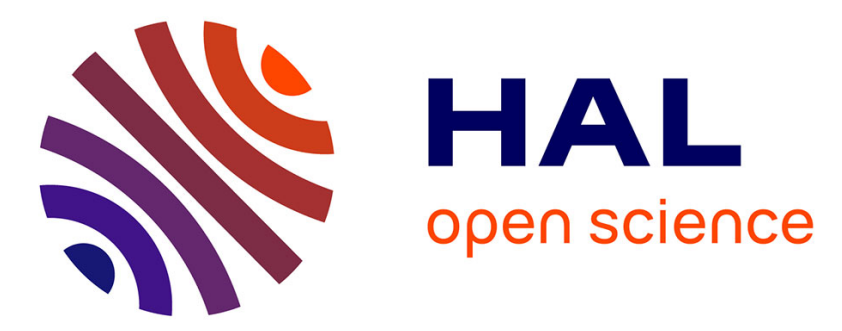

\title{
Theoretical study of a thermo-acousto-electric generator equipped with an electroacoustic feedback loop
}

Côme Olivier, Guillaume Penelet, Gaelle Poignand, Pierrick Lotton

\section{To cite this version:}

Côme Olivier, Guillaume Penelet, Gaelle Poignand, Pierrick Lotton. Theoretical study of a thermoacousto-electric generator equipped with an electroacoustic feedback loop. Internatinoal Symposium on Nonlinear Acousics, Jun 2015, Lyon, France. pp.060007, 10.1063/1.4934432 . hal-01400333

\section{HAL Id: hal-01400333 \\ https://hal.science/hal-01400333}

Submitted on 22 Nov 2018

HAL is a multi-disciplinary open access archive for the deposit and dissemination of scientific research documents, whether they are published or not. The documents may come from teaching and research institutions in France or abroad, or from public or private research centers.
L'archive ouverte pluridisciplinaire HAL, est destinée au dépôt et à la diffusion de documents scientifiques de niveau recherche, publiés ou non, émanant des établissements d'enseignement et de recherche français ou étrangers, des laboratoires publics ou privés. 


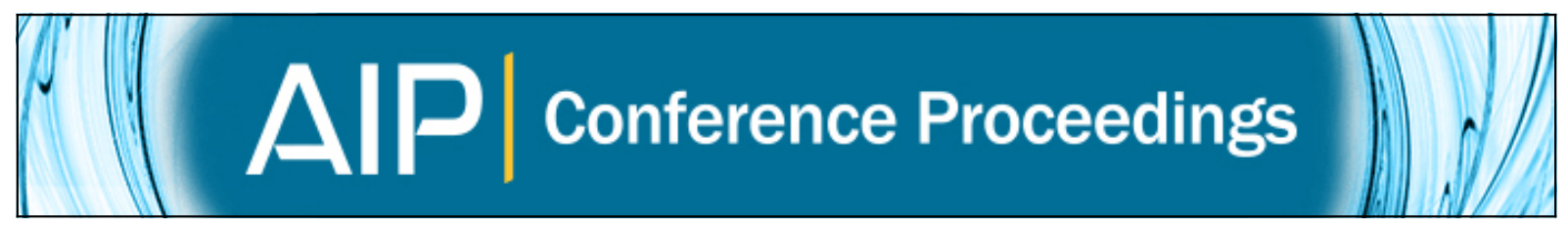

Theoretical study of a thermo-acousto-electric generator equipped with an electroacoustic feedback loop

Come Olivier, Guillaume Penelet, Gaelle Poignand, and Pierrick Lotton

Citation: AIP Conference Proceedings 1685, 060007 (2015); doi: 10.1063/1.4934432

View online: http://dx.doi.org/10.1063/1.4934432

View Table of Contents: http://scitation.aip.org/content/aip/proceeding/aipcp/1685?ver=pdfcov

Published by the AIP Publishing

\section{Articles you may be interested in}

Active control of thermoacoustic amplification in a thermo-acousto-electric engine

J. Appl. Phys. 115, 174905 (2014); 10.1063/1.4874743

Study of a thermoelectric system equipped with a maximum power point tracker for stand-alone electric generation.

AIP Conf. Proc. 1449, 520 (2012); 10.1063/1.4731608

PERFORMANCE STUDY OF LIQUID NITROGEN THERMO-SIPHON COOLING LOOP

AIP Conf. Proc. 1218, 1139 (2010); 10.1063/1.3422276

Acousto-electric generation of charged clusters and drop arrays

J. Acoust. Soc. Am. 101, 3058 (1997); 10.1121/1.418713

A study on designing the amplitude gain of the feedback loop of an electroacoustic system.

J. Acoust. Soc. Am. 90, 2239 (1991); 10.1121/1.401572 


\title{
Theoretical Study of a Thermo-Acousto-Electric Generator Equipped with an Electroacoustic Feedback loop
}

\author{
Come Olivier ${ }^{\text {a) }}$, Guillaume Penelet, Gaelle Poignand and Pierrick Lotton \\ Laboratoire d'Acoustique de l'Université du Maine UMR-CNRS 6613, Av. Olivier Messicen, 72085 Le Mans cedex9

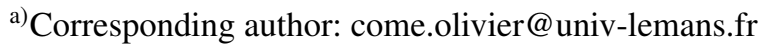

\begin{abstract}
A simplified model of a Stirling-type thermoacoustic engine coupled to a resonant mechanical system is presented. The acoustic network is presented as its temperature-dependent lumped element equivalent, and the nonlinear effects involved in such engines are accounted for in a nonlinear heat equation governing the temperature distribution through the thermoacoustic core. The low-order model is sufficient to capture the behavior of the engine, both in terms of stability and dynamic behavior.
\end{abstract}

\section{INTRODUCTION}

Thermoacoustic wave-generators are usually designed and optimized using software tools $[1,2]$ based on the linear thermoacoustic theory. These tools sometimes fail to predict accurately the operating point of a thermoacoustic engine from the balance between the thermoacoustic amplification process and the numerous nonlinear effects saturating wave amplitude growth. In fact, though they are well identified - a non exhaustive list of these effects includes (apart from the thermoacoustic heat pumping accompanying wave amplification) different kinds of streaming, nonlinear acoustic propagation or aerodynamical and thermal entrance effects - , these effects are very difficult to describe properly. They are responsible for both dissipation of acoustic power and perturbations of the temperature field in the thermoacoustic core, that together limit the overall performances of thermal-to-acoustic conversion. Therefore, the development of adequate simulation tools is still needed to describe the high level of complexity of the processes involved above the onset of thermoacoustic instability. Direct numerical simulation seems to be the only way to reproduce quantitatively the effects mentioned above, but it is still limited by large computation times inherent to the complicated physics and multiple time and space scales involved in the description of thermoacoustic engines. Simplified analytic models can help in getting a deeper physical insight about the processes involved, but they are also based on substantial approximations.

In this study, we use a simplified modeling of thermoacoustic engines to help understanding recent experimental observations dealing with the external forcing of thermoacoustic oscillations $[3,4,5]$. Indeed, at the present time the multiplicity of the involved nonlinear effects and their complexity does not allow to understand why and how the mechanisms at stake in this process of active control enable to increase the global efficiency of the thermoacoustic engine. A prerequisite is to understand deeply and to describe properly the self-sustained oscillator itself before describing its nonlinear coupling with external sound sources. A first step is to find a qualitative description of the dynamics of the system well-suited for the analysis of its bifurcation diagram and its response to external forcing. Such kind of approach is very common in nonlinear dynamics in which complicated systems are sometimes summarized by a few ordinary differential equations.

\section{MODEL}

In this study, a simplified model of a thermo-acousto-electric generator is proposed. This reduced-order model is based on a lumped element approach for the description of the acoustic propagation in the engine, coupled with a resolution of nonlinear unsteady heat transfer through the thermoacoustic core based on a finite difference scheme. 



FIGURE 1. Thermo-acousto-electric generator (TAEG) equipped with an electroacoustic feedback loop. The resonator and alternator have been reduced down to a coupled mechanical damped oscillator. (a): Schematic view; (b): Equivalent electrical circuit. The amplification gain $\Upsilon=s G$ is the product of the pressure measuring system sensibility $s$ and voltage amplification $G$.

\section{Acoustic network}

Under reasonable assumptions for quasi-traveling wave engines (long wavelength, distributed losses negligible towards regenerator losses, quasi-isothermal thermodynamic cycle, perfect heat exchangers, and quasi 1-D acoustic and temperature distributions along the $x$-direction), the acoustic network of the looped engine (in black) coupled to a resonating system (in blue), as represented schematically in Figure 1-(a), can be approximated by the equivalent electrical circuit given in Figure 1-(b). The behavior of the equivalent circuit is to be expressed as an equivalent linear system of 17 coupled first-order ODEs relating the acoustic pressures $p$ and volumic flow rates $u$ handled in the matrix form

$$
\mathrm{d}_{t} \mathbf{x}=\mathcal{M}(t) \mathbf{x}(t)
$$

where $\mathbf{x}$ is the state-vector, and $\mathcal{M}$ the Jacobian matrix of the system. The amplification gain $g$ of the regenerator, is controlled by temperature distribution $T(x)$ in the core, answering to a nonlinear heat equation which accounts for acoustically induced heat transport.

Recent proof of concept studies [3,4] performed at LAUM have already shown that the external forcing of thermoacoustic oscillations could be an interesting way to increase the efficiency of a thermoacoustic prime-mover. In particular, we have shown that the adjunction of an adequately tuned electroacoustic feedback loop to a thermoacousto-electric transducer can lead to the increase of its global efficiency [4]. Using a reference pressure signal from the engine as an input, an auxiliary acoustical source (in green in Figure 1) is used to control self-sustained thermoacoustic oscillations in the engine. By tuning the properties of this feedback (in terms of amplification gain $G$ and phase-shift $\Phi$ relative to the reference signal, in orange in Figure 1), the efficiency of the transducer can either be reduced down to the death of the auto-oscillations, or increased, thus generating a greater output power for the same heat input.

\section{Heat transfer}

The temperature distribution $T_{0}(t, x)$ in the thermoacoustic core is described with a nonlinear heat equation which accounts for thermoacoustic heat pumping, and when necessary other acoustically induced heat transport, coupled with boundary conditions assuring heat flow and temperature continuity, as well as the heat input in the HHX. The thermal problem is discretized, both in time and space by a nodal approach, so that the temperature $\hat{T}$ is described by a first order ODE system [6], related to the discretized heat distribution $\hat{Q}$

$$
\mathrm{d}_{t} \hat{T}=\mathcal{N}(t) \hat{T}+\mathcal{P}(t) \hat{Q}
$$




\section{RESULTS}

\section{Stability}

The Jacobian matrix $\mathcal{M}(t)$ allows for an evaluation of the stability of the system. Indeed, if all its eigenvalues are with negative real part, the system is asymptotically stable. If it has one conjugate couple of eigenvalues $\lambda_{k}$ with a real positive part, it is asymptotically unstable with an amplification rate $\sigma=\mathfrak{R}\left[\lambda_{k}\right]$ and frequency of oscillation $f_{o}=$ $2 \pi\left|\mathfrak{J}\left[\lambda_{k}\right]\right|$. The change of sign of $\mathfrak{R}\left[\lambda_{k}\right]$ gives therefore direct access to the onset conditions, in term of temperature gradient and frequency of the instability as a function of the feedback loop parameters, as shown in Figure 2.
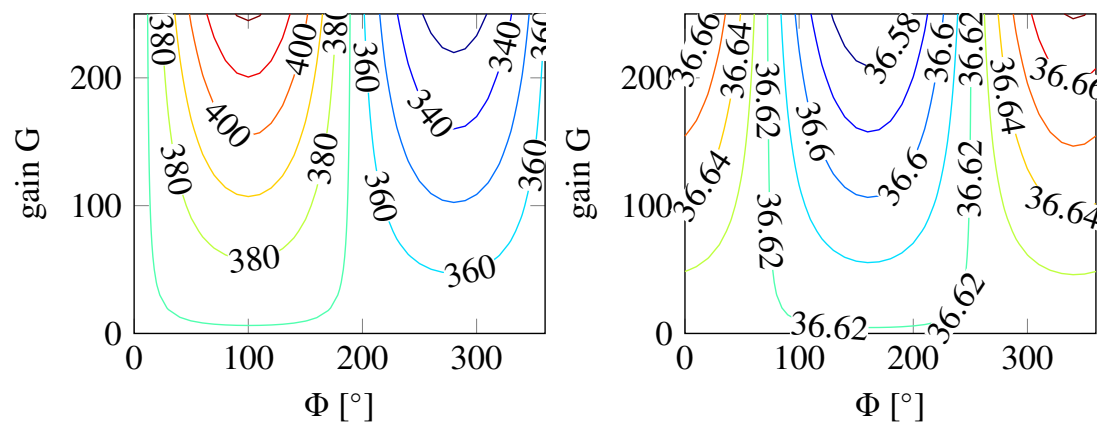

FIGURE 2. Predicted onset conditions under the influence of the feedback loop. (a) Temperature difference; (b) Frequency of the instability at onset. The experimental onset threshold gives $\Delta T_{o}(G=0, \Phi=0)=374.5 \mathrm{~K}, f_{o}(G=0, \Phi=0) \simeq 40.2 \mathrm{~Hz}$.

\section{Influence of the electroacoustic feedback}

The influence of the electroacoustic feedback loop on the system is studied in steady-state condition. The state of the engine is searched in the form of the solution of the nonlinear system, with $\mathbf{X}$ representing the amplitudes of the state vector,

$$
\begin{aligned}
\mathrm{d}_{t} \mathbf{X}(\hat{T}, G, \Phi) & =0, \\
\mathrm{~d}_{t} \hat{T}\left(x, \mathbf{X}, \dot{Q}_{h}\right) & =0,
\end{aligned}
$$

that is, for a given set of control parameters $\left(\dot{Q}_{h}, G, \Phi\right)$, the state for which thermal equilibrium is reached in the thermoacoustic core (the temperature distribution does change either in amplitude or in shape) and the amplitude of acoustic variable is saturated.

In order to determine the influence of the different nonlinear effects in the observed behavior, a solution is searched at first accounting only for thermoacoustic heat pumping, and considering that the thermoacoustic core is thermally isolated $(\gamma=0, \overline{\mathbf{V}}=0)$. The computed and experimentally determined steady-state under the influence of acoustic feedback are shown on Figure 3.

Though a good qualitative agreement is found, allowing to render the phases and amplification gain range for which the oscillations stall as well as the efficiency improvement on the opposite phase range, the amplitude of the induced efficiency variations and the temperature reached during oscillation death suggest that more phenomena are to be accounted for a more truthful rendering of the experimental behavior.

\section{CONCLUSIONS}

A simplified model of a Stirling thermoacoustic engine has been presented, consisting of a lumped element representation of the acoustic network coupled with a nonlinear heat equation. The first results provided by the model show a good agreement with the experimental data for the qualification of the stability of the system. The nonlinear behavior observed under the influence of an electroacoustic feedback loop (improvement of the efficiency, oscillation death)are -at least qualitatively - transcribed. The model will be extended to capture more complicated behavior (hysteresis of the stability threshold) and the results interpreted to gain a physical insight of the phenomena at stake in the control of the performances. 

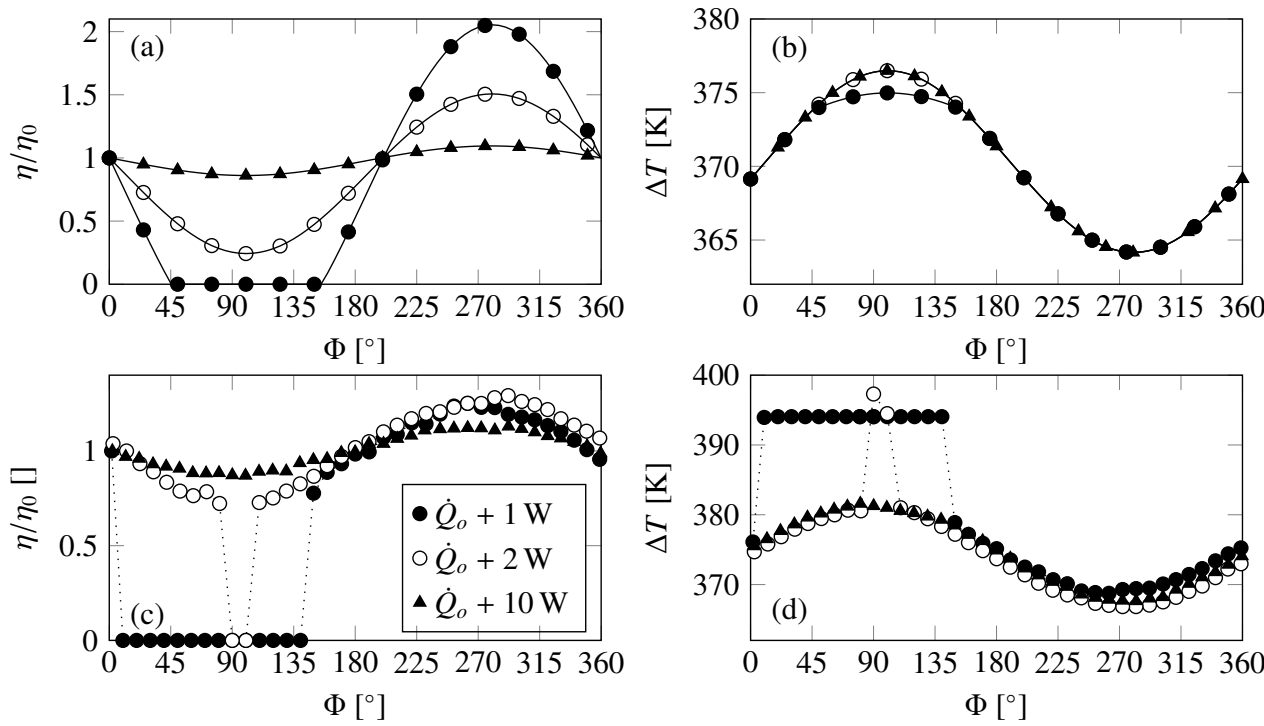

FIGURE 3. (a)\&(c) Global efficiency $\eta$ as a function of the phase-shift $\Phi$ for various heat input above onset $\dot{Q}_{h}>\dot{Q}_{o}$ and $G=32$ (b) \&(d) Corresponding temperature difference $\Delta T$ in the regenerator. (a-b) Predicted and (c-d) Experimental. A zero-efficiency point mean oscillation death for that set of control parameters.

\section{REFERENCES}

1. W. C. Ward and G. W. Swift. Design Environment for Low-Amplitude Thermoacoustic Engines. J. Acoust. Soc. Am., 95(6):3671-3672, 1994.

2. M. Guedra and G. Penelet. On the use of a complex frequency for the description of thermoacoustic engines. Acta Acust. united Ac., 98(2):232-241, 2012.

3. C. Desjouy, G. Penelet, and P. Lotton. Active control of thermoacoustic amplification in an annular engine. $J$. Appl. Phys., 108:114904, 2010.

4. C. Olivier, G. Penelet, G. Poignand, and P. Lotton. Active control of thermoacoustic amplification in a thermoacousto-electric engine. J. Appl. Phys., 115:174905, 2014.

5. G. Poignand, C. Olivier, G. Penelet, and P. Lotton. Hysteretic behaviour induced by an electroacoustic feedback loop in a thermo-acousto-electric generator. subm. to Appl. Acoust., 2015.

6. P. Lagonotte, Y. Bertin, and J.-B. Saulnier. Analyse de la qualité de modèles nodaux réduits à l'aide de la méthode des quadripôles. Int. J. Therm. Sci., 38(1):51-65, 1999. 